\title{
An input coupler for a W-band gyro-TWA
}

\author{
Liang Zhang, Wenlong He, Craig R. Donaldson, Kevin Ronald, Paul McElhinney and \\ Adrian W. Cross \\ Department of Physics, SUPA, University of Strathclyde \\ Glasgow, Scotland, UK, G4 ONG, liang.zhang@strath.ac.uk
}

\begin{abstract}
The input coupler that is used to launch the desired wave into the interaction region is an important component for a gyro-TWA. In this paper, a side-wall rectangular-to-circular coupler aiming to achieve a high coupling coefficient was designed for a W-band gyroTWA. A broadband reflector was optimized to take the place of the cut-off waveguide to allow a much bigger beam tunnel to reduce the alignment difficulty.
\end{abstract}

Keywords: rectangular-to-circular coupler, T-junction.

\section{Introduction}

The gyro-BWO and gyro-TWA are known as sources of frequency-tunable high-frequency high-power coherent radiation. They can be used in applications such as highresolution radar, plasma heating, electron paramagnetic resonance spectroscopy, and deep-space communication. Research of gyro-BWO/TWAs based on a helically corrugated waveguide (HCW) [1] demonstrated excellent results in achieving high-power and widefrequency tunability. A W-band gyro-BWO with a 3fold helically corrugated interaction waveguide was measured to have maximum output power of $12 \mathrm{~kW}$ when driven by a $40 \mathrm{kV}, 1.5 \mathrm{~A}$, annular-shaped largeorbit electron beam. The frequency tuning band is $88-$ $102.5 \mathrm{GHz}$ by adjusting the cavity magnetic field [2 - 4]. A W-band gyro-TWA is currently being experimentally studied following the gyro-BWO experiments. It shares most of the components with the gyro-BWO, such as the cusp electron gun [5], the solenoids, the polarizer [6], the input coupler and the multiple-layer microwave window [7]. The output $\mathrm{TE}_{11}$ mode of the gyro-TWA will be converted into a Gaussian beam by using a corrugated horn [8]. In the simulation, it was predicted to achieve an output power of $5 \mathrm{~kW}$ in the frequency tuning band of 90-100 GHz.

Figure 1 shows the schematic of the gyro-TWA. The input microwave signal is coupled into the system between the cusp electron gun and the interaction region, and the amplified microwaves are coupled out downstream through a window. An efficient coupler therefore plays a very important role in the performance of the gyro-TWA. In this paper, a broadband side-wall coupler which is used to convert the $\mathrm{TE}_{10}$ mode of the rectangular waveguide to the $\mathrm{TE}_{11}$ mode in the circular waveguide is presented. The operating frequency range is $84-104 \mathrm{GHz}$. It was preliminarily designed for the $\mathrm{W}$ band gyro-BWO and covered the operating frequency range of the gyro-TWA (90-100 GHz).

\section{Input coupler}

The simplest side-wall coupler can be made of a circular-to-rectangular T-junction, as shown in Figure 2. The injected $\mathrm{TE}_{10}$ mode from port 2 can be efficiently converted into a $\mathrm{TE}_{11}$ mode at port 1 and 3 . The scattering parameters of the rectangular-to-circular $\mathrm{T}$ junction can be easily calculated by finite-difference time-domain method (FDTD) or mode matching method.

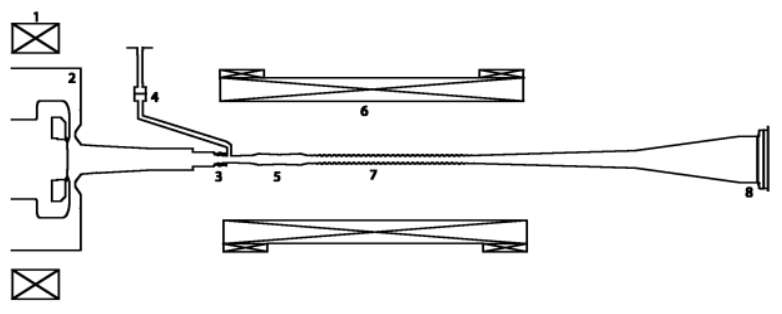

Figure 1. Experimental setup of the gyro-TWA.

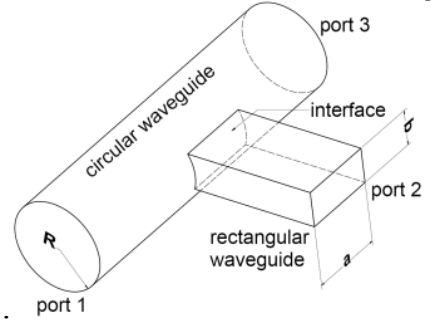

Figure 2. Rectangular-to-circular T-junction.

Parameter sweeping can be used to determine the dimensions of $a$ and $b$ to get the maximum coupling coefficient over port 2 and 1 if the circular waveguide radius $R$ is fixed. For example, $a=1.88 \mathrm{~mm}$ and $b=0.98 \mathrm{~mm}$ to get the maximum S21 over the frequency range $84-104 \mathrm{GHz}$ when $R=1.25 \mathrm{~mm}$. However, the coupling coefficient is only about $-4 \mathrm{~dB}$ even with the optimal dimensions. It is not good enough. In the practical design, a cut-off waveguide section was applied to port 3 to enhance the coupling coefficient between port 1 and 2, as shown in Figure 3.

The coupling coefficients of the T-junction connecting with a cut-off waveguide in different radii $R c$ and lengths $L$ were simulated by CST Microwave Studio and the results are shown in Figure 4. It shows that $S_{21}$ improves as $R c$ is reduced when $L=0$. It is obvious because the electromagnetic wave stops propagating to port 3 and it is either reflected back to port 2 or travels to port 1 to improve the coupling coefficient. The higher reflection caused by the circular waveguide step, the higher coupling coefficient it can achieve. The waveguide short has a unity reflectivity in the whole frequency range and achieves the best transmission coefficient. The bandwidth of the coupler, which is defined by $-1 \mathrm{~dB}$ coupling coefficient, increases as the length of $L$ is reduced when a waveguide short is connected $(R c=0)$. As the waveguide short has unity reflectivity, the only parameter that can affect the bandwidth is the phase response of the waveguide with length $L$. The longer the length $L$ is, the larger phase 
response difference in the operating frequency range will be, and the narrower bandwidth the coupler will have.

A useful conclusion from the simulations is, in order to achieve a large bandwidth, the reflection in the port 3 side needs to be as high as possible and at the same time, the phase response difference needs to be as small as possible.

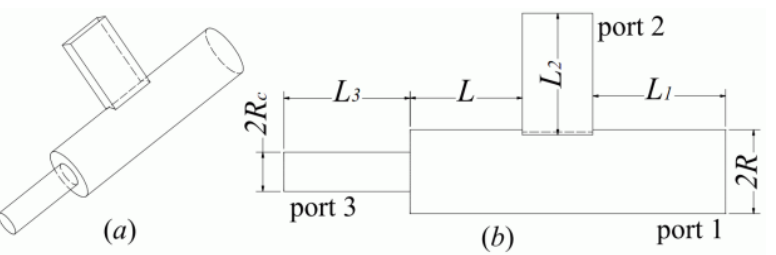

Figure 3. T-junction with cut-off waveguide, (a) 3D view, (b) front view.

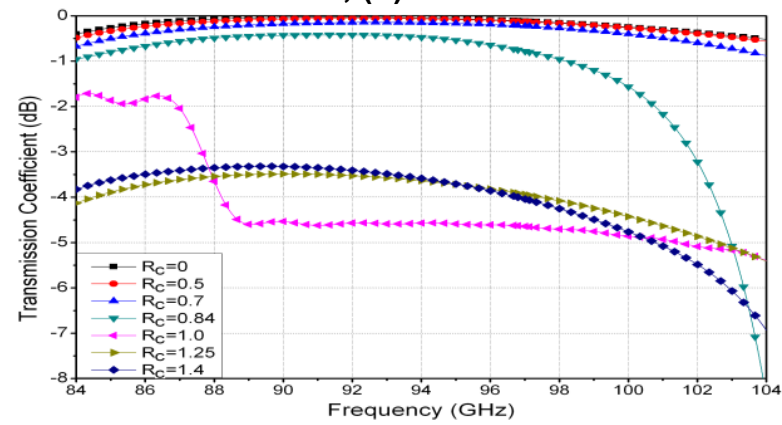

(a) $(L=0$, unit for $R c$ is $\mathrm{mm})$

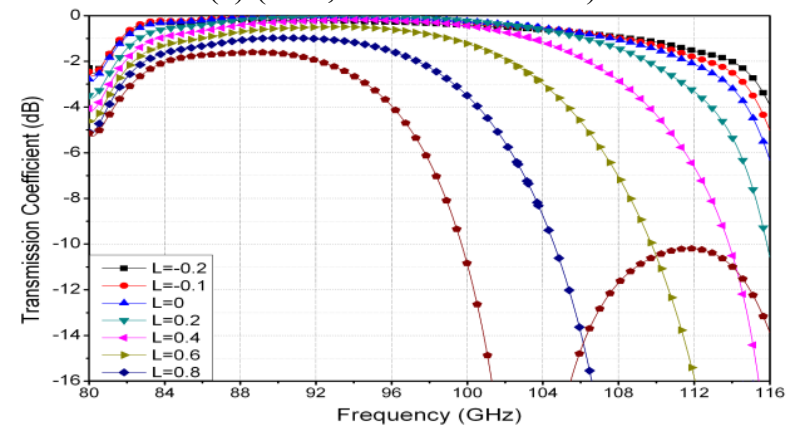

(b) $(R c=0$, unit for $L$ is $\mathrm{mm})$

Fig. 4 The transmission coefficient when (a) $L=0$, (b) $R c=0$.

The cut-off waveguide is a good solution for low frequencies. However, in the high frequency band, the radius of the cut-off section is rather small, such as $0.8 \mathrm{~mm}$ to cut-off the $104 \mathrm{GHz}$ radiation. The beam tunnel is quite close to the electron beam and adds to the difficulty of alignment. In our design, a broadband reflector was optimized to take the place of the cut-off waveguide. The reflector is able to be overmoded therefore it is able to have a much larger radius. The concept was derived from the Bragg reflector, which has excellent mode-selection reflection in a narrow bandwidth. Our previous study proved that a broad bandwidth can be achieved by changing the corrugation profiles of the Bragg structures [9]. By taking the phase response into account in the optimization, and using the conclusion in the last section as the goal of the design, a broadband reflector was optimized to achieve a $-1 \mathrm{~dB}$ coupling coefficient over a $17.5 \mathrm{GHz}$ bandwidth. To achieve a $20 \mathrm{GHz}$ bandwidth, the whole structure can be scaled down by a factor of 0.96 , which means from waveguide radius $1.25 \mathrm{~mm}$ down to $1.20 \mathrm{~mm}$. The optimized input coupler as well as the simulated coupling coefficient is shown in Figure 5.

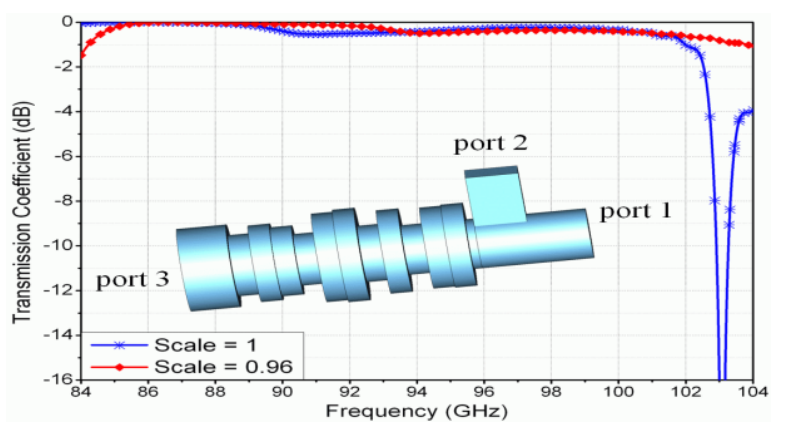

Fig. 5 The performance of the designed coupler.

\section{Acknowledgements}

The authors would like to thank EPSRC and STFC UK for supporting this work.

\section{References}

1. L. Zhang, W. He, K. Ronald, A.D.R. Phelps, et al., "Multi-mode coupling wave theory for helically corrugated waveguide," IEEE Trans. Microw. Theory Tech. 60, pp. 1-7, 2012.

2. W. He, C.R. Donaldson, F. Li, L. Zhang, et al., "Wband gyro-devices using helically corrugated waveguide and cusp gun: design, simulation and experiment," TST 4, pp. 9-19, 2011.

3. W. He, C.R. Donaldson, L. Zhang, K. Ronald, et al., "Numerical simulation of a gyro-BWO with a helically corrugated interaction region, cusp electron gun and depressed collector," ISBNL 978953-307-620-1. InTech.

4. W. He, C.R. Donaldson, L. Zhang, K. Ronald, et al., "High power wideband gyrotron backward wave oscillator operating towards the terahertz region," Phys. Rev. Lett. 110, 165101, 2013.

5. C. R. Donaldson, W. He, A.W. Cross, F. Li, et al., "A cusp electron gun for millimeter wave gyrodevices", Appl. Phys. Lett., 96, 141501, 2010.

6. L. Zhang, C.R. Donaldson and W. He, "Design and measurement of a polarization converter based on a truncated circular waveguide," J. Phys. D: Appl. Phys. 45, 345103, 2012.

7. C.R. Donaldson, W. He, L. Zhang, and A.W. Cross, "A W-band multi-layer microwave window for pulsed operation of gyro-devices," IEEE Microw. Wireless Compon. Lett., 23, pp. 237-239, 2013.

8. P. McElhinney, C.R. Donaldson, L. Zhang, and W. $\mathrm{He}$, "A high directivity broadband corrugated horn for W-band gyro-devices," IEEE Trans. Antennas Propag. 61, pp. 1453-1456, 2013.

9. L. Zhang, W. He, A.W. Cross, A.D.R. Phelps, et al., "Design of an energy recovery system for a gyrotron backward-wave oscillator," IEEE Trans. Plasma Sci. 37, pp. 309-394, 2009. 